\title{
Identities on poly-Dedekind sums
}

Taekyun Kim', Dae San Kim², Hyunseok Lee ${ }^{1}$ and Lee-Chae Jang ${ }^{3 *}$

${ }^{\text {"Correspondence: }}$

Icjang@konkuk.ac.kr

${ }^{3}$ Graduate School of Education, Konkuk University, Seoul, Republic of Korea

Full list of author information is available at the end of the article

\begin{abstract}
Dedekind sums occur in the transformation behavior of the logarithm of the Dedekind eta-function under substitutions from the modular group. In 1892, Dedekind showed a reciprocity relation for the Dedekind sums. Apostol generalized Dedekind sums by replacing the first Bernoulli function appearing in them by any Bernoulli functions and derived a reciprocity relation for the generalized Dedekind sums. In this paper, we consider the poly-Dedekind sums obtained from the Dedekind sums by replacing the first Bernoulli function by any type 2 poly-Bernoulli functions of arbitrary indices and prove a reciprocity relation for the poly-Dedekind sums.
\end{abstract}

MSC: 11F20; 11B68; 11B83

Keywords: Poly-Dedekind sum; Polyexponential function; Type 2 poly-Bernoulli polynomial

\section{Introduction}

To give concise definition of the Dedekind sums, we introduce the notation

$$
((x))=\left\{\begin{array}{ll}
x-[x]-\frac{1}{2} & \text { if } x \notin \mathbb{Z}, \\
0 & \text { if } x \in \mathbb{Z},
\end{array} \quad(\text { see }[1,4])\right.
$$

where $[x]$ denotes the greatest integer not exceeding $x$.

It is well known that the Dedekind sums are defined by

$$
S(h, m)=\sum_{\mu=1}^{m}\left(\left(\frac{\mu}{m}\right)\right)\left(\left(\frac{h \mu}{m}\right)\right) \quad(\text { see }[1,4,6-8,11-13])
$$

where $h$ is any integer.

From (2) we note that

$$
S(h, m)=\sum_{\mu=1}^{m}\left(\frac{\mu}{m}-\frac{1}{2}\right)\left(\left(\frac{h \mu}{m}\right)\right)=\sum_{\mu=1}^{m} \frac{\mu}{m}\left(\left(\frac{h \mu}{m}\right)\right) \quad(\text { see }[7,8])
$$

As is well known, the Bernoulli polynomials are given by

$$
\frac{t}{e^{t}-1} e^{x t}=\sum_{n=0}^{\infty} B_{n}(x) \frac{t^{n}}{n !} \quad(\text { see }[1-13])
$$

(c) The Author(s) 2020. This article is licensed under a Creative Commons Attribution 4.0 International License, which permits use, sharing, adaptation, distribution and reproduction in any medium or format, as long as you give appropriate credit to the original author(s) and the source, provide a link to the Creative Commons licence, and indicate if changes were made. The images or other third party material in this article are included in the article's Creative Commons licence, unless indicated otherwise in a credit line to the material. If material is not included in the article's Creative Commons licence and your intended use is not permitted by statutory regulation or exceeds the permitted use, you will need to obtain permission directly from the copyright holder. To view a copy of this licence, visit http://creativecommons.org/licenses/by/4.0/. 
When $x=0, B_{n}=B_{n}(0)(n \geq 0)$ are called the Bernoulli numbers.

From (4) we note that

$$
B_{n}(x)=\sum_{l=0}^{n}\left(\begin{array}{l}
n \\
l
\end{array}\right) B_{l} x^{n-l}=(B+x)^{n} \quad(n \geq 0)(\text { see }[2-5,7,8]),
$$

with the usual convention about replacing $B^{n}$ by $B_{n}$.

We observe that

$$
\sum_{l=0}^{n-1} e^{l t}=\frac{t}{t\left(e^{t}-1\right)}\left(e^{n t}-1\right)=\sum_{j=0}^{\infty}\left(\frac{B_{j+1}(n)-B_{j+1}}{j+1}\right) \frac{t^{j}}{j !} \quad(n \in \mathbb{N}) .
$$

Thus by (6) we get

$$
\sum_{l=0}^{n-1} l^{j}=\frac{1}{j+1}\left(B_{j+1}(n)-B_{j+1}\right) \quad(n \in \mathbb{N}, j \geq 0) .
$$

Recently, Kim and Kim [5, 9] considered the polyexponential function of index $k$ given by

$$
\operatorname{Ei}_{k}(x)=\sum_{n=1}^{\infty} \frac{x^{n}}{n^{k}(n-1) !} \quad(k \in \mathbb{Z})
$$

Note that $\operatorname{Ei}_{1}(x)=e^{x}-1$.

In [5] the type 2 poly-Bernoulli polynomials of index $k$ are defined in terms of the polyexponential function of index $k$ as

$$
\frac{\operatorname{Ei}_{k}(\log (1+t))}{e^{t}-1} e^{x t}=\sum_{n=0}^{\infty} B_{n}^{(k)}(x) \frac{t^{n}}{n !} \quad(k \in \mathbb{Z})
$$

When $x=0, B_{n}^{(k)}=B_{n}^{(k)}(0)(n \geq 0)$ are called the type 2 poly-Bernoulli numbers of index $k$. Note that $B_{n}^{(1)}(x)=B_{n}(x)$ are the Bernoulli polynomials.

The fractional part of $x$ is denoted by

$$
\langle x\rangle=x-[x] .
$$

The Bernoulli functions are defined by

$$
\bar{B}_{n}(x)=B_{n}(\langle x\rangle) \quad(n \geq 0)(\text { see }[1,4,11]) .
$$

Thus by (3) and (11) we get

$$
\begin{aligned}
S(h, m) & =\sum_{\mu=1}^{m-1} \frac{\mu}{m}\left(\frac{h \mu}{m}-\left[\frac{h \mu}{m}\right]-\frac{1}{2}\right) \\
& =\sum_{\mu=1}^{m-1} \frac{\mu}{m} \bar{B}_{1}\left(\frac{h \mu}{m}\right)=\sum_{\mu=1}^{m-1} \bar{B}_{1}\left(\frac{\mu}{m}\right) \bar{B}_{1}\left(\frac{h \mu}{m}\right),
\end{aligned}
$$

where $h, m$ are relatively prime positive integers. 
We need the following lemma, which is well-known and easily shown.

Lemma 1 Let $n$ be a nonnegative integer, and let $d$ be a positive integer. Then we have:

(a) $\sum_{i=0}^{d-1} B_{n}\left(\frac{x+i}{d}\right)=d^{1-n} B_{n}(x)$,

(b) $\sum_{i=0}^{d-1} \bar{B}_{n}\left(\frac{x+i}{d}\right)=d^{1-n} \bar{B}_{n}(x)$, and

(c) $\sum_{i=0}^{d-1} B_{n}\left(\frac{(x\rangle+i}{d}\right)=\sum_{i=0}^{d-1} \bar{B}_{n}\left(\frac{x+i}{d}\right)$ for all real $x$.

Dedekind showed that the quantity $S(h, m)=\sum_{\mu=1}^{m-1} \frac{\mu}{m} \bar{B}_{1}\left(\frac{h \mu}{m}\right)$ occurs in the transformation behavior of the logarithm of the Dedekind eta-function under substitutions from the modular group. In 1892, he showed the following reciprocity relation for Dedekind sums:

$$
S(h, m)+S(m, h)=\frac{1}{12}\left(\frac{h}{m}+\frac{1}{h m}+\frac{m}{h}\right)-\frac{1}{4}
$$

if $h$ and $m$ are relatively prime positive integers.

Apostol [1] considered the generalized Dedekind sums given by

$$
S_{p}(h, m)=\sum_{\mu=1}^{m-1} \frac{\mu}{m} \bar{B}_{p}\left(\frac{h \mu}{m}\right)
$$

and showed that they satisfy the reciprocity relation

$$
\begin{aligned}
(p & +1)\left(h m^{p} S_{p}(h, m)+m h^{p} S_{p}(m, h)\right) \\
& =p B_{p+1}+\sum_{s=0}^{p+1}\left(\begin{array}{c}
p+1 \\
s
\end{array}\right)(-1)^{s} B_{s} B_{p+1-s} h^{s} m^{p+1-s} .
\end{aligned}
$$

In this paper, we consider the poly-Dedekind sums defined by

$$
S_{p}^{(k)}(h, m)=\sum_{\mu=1}^{m-1} \frac{\mu}{m} \bar{B}_{p}^{(k)}\left(\frac{h \mu}{m}\right),
$$

where $B_{p}^{(k)}(x)$ are the type 2 poly-Bernoulli polynomials of index $k$ (see (9)), and $\bar{B}_{p}^{(k)}(x)=$ $B_{p}^{(k)}(\langle x\rangle)$ are the type 2 poly-Bernoulli functions of index $k$. Note that $S_{p}^{(1)}(h, m)=S_{p}(h, m)$. We show the following reciprocity relation for the poly-Dedekind sums (see Theorem 10):

$$
\begin{aligned}
h m^{p} & S_{p}^{(k)}(h, m)+m h^{p} S_{p}^{(k)}(m, h) \\
& =\sum_{\mu=0}^{m-1} \sum_{j=0}^{p} \sum_{\nu=0}^{h-1} \sum_{l=1}^{p-j+1} \frac{(m h)^{j-1}\left(\begin{array}{c}
p \\
j
\end{array}\right) S_{1}(p-j+1, l)}{(p-j+1) l^{k-1}}\left((\mu h) m^{p-j}+(m v) h^{p-j}\right) \bar{B}_{j}\left(\frac{v}{h}+\frac{\mu}{m}\right) .
\end{aligned}
$$

For $k=1$, this reciprocity relation for the poly-Dedekind sums reduces to that for the generalized Dedekind sums given by (see Corollary 11)

$$
\begin{aligned}
h m^{p} & S_{p}(h, m)+m h^{p} S_{p}(m, h) \\
& =\sum_{\mu=0}^{m-1} \sum_{\nu=0}^{h-1}(m h)^{p-1}(\mu h+m v) \bar{B}_{p}\left(\frac{v}{h}+\frac{\mu}{m}\right) .
\end{aligned}
$$


In Sect. 2, we derive various facts about the type 2 poly-Bernoulli polynomials, which will be needed in the next section. In Sect. 3, we define the poly-Dedekind sums and demonstrate a reciprocity relation for them.

\section{On type 2 poly-Bernoulli polynomials}

Note that by (9)

$$
\begin{aligned}
\frac{\operatorname{Ei}_{k}(\log (1+t))}{e^{t}-1} e^{x t} & =\sum_{l=0}^{\infty} B_{l}^{(k)} \frac{t^{l}}{l !} \sum_{m=0}^{\infty} \frac{x^{m}}{m !} t^{m} \\
& =\sum_{n=0}^{\infty}\left(\sum_{l=0}^{n}\left(\begin{array}{l}
n \\
l
\end{array}\right) B_{l}^{(k)} x^{n-l}\right) \frac{t^{n}}{n !}
\end{aligned}
$$

Thus by (14) we get

$$
B_{n}^{(k)}(x)=\sum_{l=0}^{n}\left(\begin{array}{l}
n \\
l
\end{array}\right) B_{l}^{(k)} x^{n-l} \quad(n \geq 0) .
$$

By (15) we get

$$
\frac{d}{d x} B_{n}^{(k)}(x)=n B_{n-1}^{(k)}(x) \quad(n \geq 1)
$$

From (9) we have

$$
\begin{aligned}
\operatorname{Ei}_{k}(\log (1+t)) & =\sum_{l=0}^{\infty} B_{l}^{(k)} \frac{t^{l}}{l !}\left(e^{t}-1\right) \\
& =\sum_{n=0}^{\infty}\left(B_{n}^{(k)}(1)-B_{n}^{(k)}\right) \frac{t^{n}}{n !}=\sum_{n=1}^{\infty}\left(B_{n}^{(k)}(1)-B_{n}^{(k)}\right) \frac{t^{n}}{n !}
\end{aligned}
$$

On the other hand,

$$
\begin{aligned}
\operatorname{Ei}_{k}(\log (1+t)) & =\sum_{m=1}^{\infty} \frac{(\log (1+t))^{m}}{m^{k}(m-1) !}=\sum_{m=1}^{\infty} \frac{1}{m^{k-1}} \frac{1}{m !}(\log (1+t))^{m} \\
& =\sum_{m=1}^{\infty} \frac{1}{m^{k-1}} \sum_{n=m}^{\infty} S_{1}(n, m) \frac{t^{n}}{n !} \\
& =\sum_{n=1}^{\infty}\left(\sum_{m=1}^{n} \frac{1}{m^{k-1}} S_{1}(n, m)\right) \frac{t^{n}}{n !}
\end{aligned}
$$

where $S_{1}(n, m)$ are the Stirling numbers of the first kind.

Therefore by (17) and (18) we obtain the following theorem.

Theorem 2 For $n \geq 1$, we have

$$
B_{n}^{(k)}(1)-B_{n}^{(k)}=\sum_{m=1}^{n} S_{1}(n, m) \frac{1}{m^{k-1}} \quad(k \in \mathbb{Z}) .
$$


By Theorem 2 we get

$$
B_{n}^{(1)}-B_{n}^{(1)}=\delta_{1, n}, \quad B_{0}^{(k)}=1, \quad B_{1}^{(k)}=-1+\frac{1}{2^{k}}, \ldots,
$$

where $\delta_{n, k}$ is the Kronecker symbol.

With (16) in mind, we now compute

$$
\begin{aligned}
\left.\left(\frac{d}{d x}\right)^{s-1}\left(x B_{p}^{(k)}(x)\right)\right|_{x=1} & =\left.\sum_{l=0}^{s-1}\left(\begin{array}{c}
s-1 \\
l
\end{array}\right)\left(\left(\frac{d}{d x}\right)^{l} x\right)\left(\left(\frac{d}{d x}\right)^{s-1-l} B_{p}^{(k)}(x)\right)\right|_{x=1} \\
& =\left.\left(\frac{d}{d x}\right)^{s-1} B_{p}^{(k)}(x)\right|_{x=1}+\left.\left(\begin{array}{c}
s-1 \\
1
\end{array}\right)\left(\frac{d}{d x}\right)^{s-2} B_{p}^{(k)}(x)\right|_{x=1} \\
& =\frac{s !}{p+1}\left(\begin{array}{c}
p+1 \\
s
\end{array}\right) B_{p-s+1}^{(k)}(1)+\frac{(s-1) s !}{(p+1)(p+2)}\left(\begin{array}{c}
p+2 \\
s
\end{array}\right) B_{p-s+2}^{(k)}(1) .
\end{aligned}
$$

On the other hand, by (15) we get

$$
\begin{aligned}
\left.\left(\frac{d}{d x}\right)^{s-1}\left(x B_{p}^{(k)}(x)\right)\right|_{x=1} & =\left.\sum_{\nu=0}^{p}\left(\begin{array}{l}
p \\
v
\end{array}\right) B_{v}^{(k)}\left(\left(\frac{d}{d x}\right)^{s-1} x^{p-v+1}\right)\right|_{x=1} \\
& =\sum_{\nu=0}^{p}\left(\begin{array}{l}
p \\
v
\end{array}\right) B_{v}^{(k)}(p-v+1) \cdots(p-v-s+3) \\
& =\sum_{\nu=0}^{p}\left(\begin{array}{l}
p \\
v
\end{array}\right) \frac{s ! B_{v}^{(k)}}{p-v+2}\left(\begin{array}{c}
p-v+2 \\
s
\end{array}\right) .
\end{aligned}
$$

Therefore by (19) and (20) we obtain the following theorem.

Theorem 3 For $s, p \in \mathbb{N}$, we have

$$
\sum_{v=0}^{p}\left(\begin{array}{l}
p \\
v
\end{array}\right)\left(\begin{array}{c}
p-v+2 \\
s
\end{array}\right) \frac{B_{v}^{(k)}}{p-v+2}=\left(\begin{array}{c}
p+1 \\
s
\end{array}\right) \frac{B_{p-s+1}^{(k)}(1)}{p+1}+\frac{s-1}{p+1}\left(\begin{array}{c}
p+2 \\
s
\end{array}\right) \frac{B_{p-s+2}^{(k)}(1)}{p+2}
$$

Now we observe that

$$
\begin{aligned}
\sum_{v=0}^{p}\left(\begin{array}{l}
p \\
v
\end{array}\right)\left(\begin{array}{c}
p-v+2 \\
s
\end{array}\right) \frac{B_{v}^{(k)}}{p-v+2} & =\sum_{\nu=0}^{p-s+2} \frac{\left(\begin{array}{l}
p \\
v
\end{array}\right)\left(\begin{array}{c}
p-v+2 \\
s
\end{array}\right)}{p-v+2} B_{v}^{(k)} \\
& =\sum_{\nu=0}^{p-s+1} \frac{\left(\begin{array}{l}
p \\
v
\end{array}\right)\left(\begin{array}{c}
p-v+2 \\
s
\end{array}\right)}{p-v+2} B_{v}^{(k)}+\frac{1}{s}\left(\begin{array}{c}
p \\
s-2
\end{array}\right) B_{p-s+2}^{(k)} .
\end{aligned}
$$

Therefore by Theorem 3 and (21) we obtain the following corollary.

Corollary 4 For $s, p \in \mathbb{N}$, we have

$$
\begin{aligned}
& \sum_{v=0}^{p-s+1}\left(\begin{array}{c}
p \\
v
\end{array}\right)\left(\begin{array}{c}
p-v+2 \\
s
\end{array}\right) \frac{B_{v}^{(k)}}{p-v+2} \\
& \quad=\left(\begin{array}{c}
p+1 \\
s
\end{array}\right) \frac{B_{p-s+1}^{(k)}(1)}{p+1}+\frac{s-1}{p+1}\left(\begin{array}{c}
p+2 \\
s
\end{array}\right) \frac{B_{p-s+2}^{(k)}(1)}{p+2}-\frac{1}{s}\left(\begin{array}{c}
p \\
s-2
\end{array}\right) B_{p-s+2}^{(k)} .
\end{aligned}
$$


From (16) we have

$$
\begin{aligned}
\int_{0}^{1} x B_{p}^{(k)}(x) d x & =\left[x \frac{B_{p+1}^{(k)}(x)}{p+1}\right]_{0}^{1}-\frac{1}{p+1} \int_{0}^{1} B_{p+1}^{(k)}(x) d x \\
& =\frac{B_{p+1}^{(k)}(1)}{p+1}-\frac{1}{p+1}\left[\frac{1}{p+2} B_{p+2}^{(k)}(x)\right]_{0}^{1} \\
& =\frac{B_{p+1}^{(k)}(1)}{p+1}-\frac{B_{p+2}^{(k)}(1)}{(p+1)(p+2)}+\frac{B_{p+2}^{(k)}}{(p+1)(p+2)} .
\end{aligned}
$$

On the other hand, by (15) we get

$$
\begin{aligned}
\int_{0}^{1} x B_{p}^{(k)}(x) d x & =\sum_{s=0}^{p}\left(\begin{array}{l}
p \\
s
\end{array}\right) B_{s}^{(k)} \int_{0}^{1} x^{p-s+1} d x \\
& =\sum_{s=0}^{p}\left(\begin{array}{l}
p \\
s
\end{array}\right) B_{s}^{(k)} \frac{1}{p+2-s} .
\end{aligned}
$$

Therefore by (22) and (23) we obtain the following theorem.

Theorem 5 For $p \in \mathbb{N}$, we have

$$
\sum_{s=0}^{p}\left(\begin{array}{l}
p \\
s
\end{array}\right) B_{s}^{(k)} \frac{1}{p+2-s}=\frac{B_{p+1}^{(k)}(1)}{p+1}-\frac{B_{p+2}^{(k)}(1)}{(p+1)(p+2)}+\frac{B_{p+2}^{(k)}}{(p+1)(p+2)}
$$

\section{Poly-Dedekind sums}

Apostol considered the generalized Dedekind sums given by

$$
S_{p}(h, m)=\sum_{\mu=1}^{m-1}(\mu / m) \bar{B}_{p}(h \mu / m) \quad(h, m, p \in \mathbb{N})
$$

where $\bar{B}_{p}(h \mu / m)=B_{p}(\langle h \mu / m\rangle)$.

Note that, for any relatively prime positive integers $h, m$, we have

$$
\begin{aligned}
S_{1}(h, m) & =\sum_{\mu=1}^{m-1}(\mu / m) \bar{B}_{1}(h \mu / m) \\
& =\sum_{\mu=1}^{m-1}((\mu / m))((h \mu / m))=S(h, m) .
\end{aligned}
$$

In this section, we consider the poly-Dedekind sums given by

$$
S_{p}^{(k)}(h, m)=\sum_{\mu=1}^{m-1}(\mu / m) \bar{B}_{p}^{(k)}(h \mu / m)
$$

where $h, m, p \in \mathbb{N}, k \in \mathbb{Z}$, and $\bar{B}_{p}^{(k)}(x)=B_{p}^{(k)}(\langle x\rangle)$ are the type 2 poly-Bernoulli functions of index $k$. 
Note that

$$
S_{p}^{(1)}(h, m)=\sum_{\mu=1}^{m-1}(\mu / m) \bar{B}_{p}(h \mu / m)=S_{p}(h, m) .
$$

Assume now that $h=1$. Then we have

$$
\begin{aligned}
S_{p}^{(k)}(1, m) & =\sum_{\mu=1}^{m-1}(\mu / m) \bar{B}_{p}^{(k)}(\mu / m) \\
& =\sum_{\mu=1}^{m-1}(\mu / m) \sum_{\nu=0}^{p}\left(\begin{array}{l}
p \\
v
\end{array}\right) B_{v}^{(k)}(\mu / m)^{p-v} \\
& =\sum_{v=0}^{p}\left(\begin{array}{l}
p \\
v
\end{array}\right) B_{v}^{(k)} m^{-(p-v+1)} \sum_{\mu=1}^{m-1} \mu^{p+1-v} \\
& =\sum_{v=0}^{p}\left(\begin{array}{l}
p \\
v
\end{array}\right) B_{v}^{(k)} m^{-(p+1-v)} \frac{1}{p+2-v}\left(B_{p+2-v}(m)-B_{p+2-v}\right) .
\end{aligned}
$$

From (5) we have

$$
\begin{aligned}
B_{p+2-v}(m)-B_{p+2-v} & =\sum_{i=0}^{p+2-v}\left(\begin{array}{c}
p+2-v \\
i
\end{array}\right) B_{i} m^{p+2-v-i}-B_{p+2-v} \\
& =\sum_{i=0}^{p+1-v}\left(\begin{array}{c}
p+2-v \\
i
\end{array}\right) B_{i} m^{p+2-v-i} .
\end{aligned}
$$

By (26) and (27) we get

$$
\begin{aligned}
S_{p}^{(k)}(1, m) & =\sum_{v=0}^{p}\left(\begin{array}{l}
p \\
v
\end{array}\right) B_{v}^{(k)} m^{-(p+1-v)} \frac{1}{p+2-v} \sum_{i=0}^{p+1-v}\left(\begin{array}{c}
p+2-v \\
i
\end{array}\right) B_{i} m^{p+2-v-i} \\
& =\frac{1}{m^{p}} \sum_{v=0}^{p}\left(\begin{array}{l}
p \\
v
\end{array}\right) \frac{B_{v}^{(k)}}{p+2-v} \sum_{i=0}^{p+1-v}\left(\begin{array}{c}
p+2-v \\
i
\end{array}\right) B_{i} m^{p+1-i} .
\end{aligned}
$$

Now we assume that $p \geq 3$ is an odd positive integer, so that $B_{p}=0$. Then we have

$$
\begin{aligned}
m^{p} S_{p}^{(k)}(1, m) & =\sum_{v=0}^{p}\left(\begin{array}{l}
p \\
v
\end{array}\right) \frac{B_{v}^{(k)}}{p+2-v} \sum_{i=0}^{p+1-v}\left(\begin{array}{c}
p+2-v \\
i
\end{array}\right) B_{i} m^{p+1-i} \\
& =\sum_{v=0}^{p}\left(\begin{array}{l}
p \\
v
\end{array}\right) \frac{B_{v}^{(k)}}{p+2-v} m^{p+1}+\sum_{v=0}^{p}\left(\begin{array}{c}
p \\
v
\end{array}\right) \frac{B_{v}^{(k)}}{p+2-v} \sum_{i=1}^{p+1-v}\left(\begin{array}{c}
p+2-v \\
i
\end{array}\right) B_{i} m^{p+1-i} \\
& =\sum_{v=0}^{p}\left(\begin{array}{l}
p \\
v
\end{array}\right) \frac{B_{v}^{(k)}}{p+2-v} m^{p+1}+\sum_{i=1}^{p+1} \sum_{v=0}^{p+1-i}\left(\begin{array}{c}
p \\
v
\end{array}\right)\left(\begin{array}{c}
p+2-v \\
i
\end{array}\right) \frac{B_{v}^{(k)}}{p+2-v} B_{i} m^{p+1-i} \\
& =\sum_{v=0}^{p}\left(\begin{array}{l}
p \\
v
\end{array}\right) \frac{B_{v}^{(k)}}{p+2-v} m^{p+1}+\sum_{i=1}^{p-1} \sum_{v=0}^{p+1-i}\left(\begin{array}{c}
p \\
v
\end{array}\right)\left(\begin{array}{c}
p+2-v \\
i
\end{array}\right) \frac{B_{v}^{(k)}}{p+2-v} B_{i} m^{p+1-i}
\end{aligned}
$$




$$
\begin{aligned}
& +\frac{1}{p+2}\left(\begin{array}{c}
p+2 \\
p+1
\end{array}\right) B_{p+1}+\sum_{v=0}^{1}\left(\begin{array}{c}
p \\
v
\end{array}\right)\left(\begin{array}{c}
p+2-v \\
p
\end{array}\right) \frac{B_{v}^{(k)}}{p+2-v} B_{p} m \\
= & \sum_{\nu=0}^{p}\left(\begin{array}{l}
p \\
v
\end{array}\right) \frac{B_{v}^{(k)}}{p+2-v} m^{p+1}+\sum_{i=1}^{p-1} \sum_{\nu=0}^{p+1-i}\left(\begin{array}{l}
p \\
v
\end{array}\right) \frac{\left(\begin{array}{c}
p+2-v \\
i
\end{array}\right)}{p+2-v} B_{v}^{(k)} B_{i} m^{p+1-i}+B_{p+1} .
\end{aligned}
$$

Therefore by (29) we obtain the following proposition.

Proposition 6 Let $p \geq 3$ be an odd positive integer. Then we have

$$
\begin{aligned}
m^{p} S_{p}^{(k)}(1, m)= & \sum_{\nu=0}^{p}\left(\begin{array}{l}
p \\
v
\end{array}\right) \frac{B_{v}^{(k)}}{p+2-v} m^{p+1}+\sum_{i=1}^{p-1} \sum_{\nu=0}^{p+1-i}\left(\begin{array}{l}
p \\
v
\end{array}\right)\left(\begin{array}{c}
p+2-v \\
i
\end{array}\right) \frac{B_{v}^{(k)}}{p+2-v} B_{i} m^{p+1-i} \\
& +B_{p+1}
\end{aligned}
$$

We still assume that $p \geq 3$ is an odd positive integer, so that $B_{p}=0$. Then from Corollary 4, Theorem 5, and Proposition 6 we note that

$$
\begin{aligned}
m^{p} S_{p}^{(k)}(1, m) & \\
= & \sum_{\nu=0}^{p}\left(\begin{array}{l}
p \\
v
\end{array}\right) \frac{B_{v}^{(k)}}{p+2-v} m^{p+1}+\sum_{i=1}^{p-1} \sum_{v=0}^{p+1-i}\left(\begin{array}{c}
p \\
v
\end{array}\right)\left(\begin{array}{c}
p+2-v \\
i
\end{array}\right) \frac{B_{v}^{(k)}}{p+2-v} B_{i} m^{p+1-i}+B_{p+1} \\
= & \left(\frac{B_{p+1}^{(k)}(1)}{p+1}-\frac{B_{p+2}^{(k)}(1)}{(p+1)(p+2)}+\frac{B_{p+2}^{(k)}}{(p+1)(p+2)}\right) m^{p+1}+B_{p+1} \\
& +\sum_{i=1}^{p-1}\left(\left(\begin{array}{c}
p+1 \\
i
\end{array}\right) \frac{B_{p+1-i}^{(k)}(1)}{p+1}+\frac{(i-1)}{(p+1)(p+2)}\left(\begin{array}{c}
p+2 \\
i
\end{array}\right) B_{p+2-i}^{(k)}(1)\right. \\
& \left.-\left(\begin{array}{c}
p \\
i-2
\end{array}\right) \frac{1}{i} B_{p+2-i}^{(k)}\right) B_{i} m^{p+1-i} .
\end{aligned}
$$

To proceed further, we note that $\left(\begin{array}{c}p \\ i-2\end{array}\right) \frac{p+1}{i}=\frac{1}{p+2}\left(\begin{array}{c}p+2 \\ i\end{array}\right)(i-1)$ for $i \geq 1$ and that $B_{1}^{(k)}(1)-B_{1}^{(k)}=1$ by Theorem 2 . Then from (30) we see that

$$
\begin{aligned}
(p+1) m^{p} S_{p}^{(k)}(1, m)= & \left(B_{p+1}^{(k)}(1)-\frac{B_{p+2}^{(k)}(1)}{p+2}+\frac{B_{p+2}^{(k)}}{p+2}\right) m^{p+1} \\
& +\sum_{i=1}^{p-1}\left(\begin{array}{c}
p+1 \\
i
\end{array}\right) B_{i} B_{p+1-i}^{(k)}(1) m^{p+1-i}+(p+1) B_{p+1} \\
& +\frac{1}{p+2} \sum_{i=1}^{p-1}\left(\begin{array}{c}
p+2 \\
i
\end{array}\right)(i-1) B_{i} B_{p+2-i}^{(k)}(1) m^{p+1-i} \\
& -\sum_{i=1}^{p-1}\left(\begin{array}{c}
p \\
i-2
\end{array}\right) \frac{(p+1)}{i} B_{p+2-i}^{(k)} B_{i} m^{p+1-i} \\
= & m^{p+1} B_{p+1}^{(k)}(1)+\sum_{i=1}^{p-1}\left(\begin{array}{c}
p+1 \\
i
\end{array}\right) B_{i} m^{p+1-i} B_{p+1-i}^{(k)}(1)+B_{p+1} \\
& +\frac{1}{p+2}(-1) m^{p+1}\left(B_{p+2}^{(k)}(1)-B_{p+2}^{(k)}\right)
\end{aligned}
$$




$$
\begin{aligned}
& +\frac{1}{p+2} \sum_{i=1}^{p-1}\left(\begin{array}{c}
p+2 \\
i
\end{array}\right)(i-1) B_{i} m^{p+1-i}\left(B_{p+2-i}^{(k)}(1)-B_{p+2-i}^{(k)}\right) \\
& +p B_{p+1} \\
& =\sum_{i=0}^{p+1}\left(\begin{array}{c}
p+1 \\
i
\end{array}\right) B_{i} m^{p+1-i} B_{p+1-i}^{(k)}(1) \\
& +\frac{1}{p+2} \sum_{i=0}^{p+1}\left(\begin{array}{c}
p+2 \\
i
\end{array}\right)(i-1) B_{i} m^{p+1-i}\left(B_{p+2-i}^{(k)}(1)-B_{p+2-i}^{(k)}\right) .
\end{aligned}
$$

Therefore by (31) we obtain the following theorem.

Theorem 7 For $m \in \mathbb{N}$ and any odd positive integer $p \geq 3$, we have

$$
\begin{aligned}
(p+1) m^{p} S_{p}^{(k)}(1, m) & \\
= & \sum_{i=0}^{p+1}\left(\begin{array}{c}
p+1 \\
i
\end{array}\right) B_{i} m^{p+1-i} B_{p+1-i}^{(k)}(1) \\
& +\frac{1}{p+2} \sum_{i=0}^{p+1}\left(\begin{array}{c}
p+2 \\
i
\end{array}\right)(i-1) B_{i} m^{p+1-i}\left(B_{p+2-i}^{(k)}(1)-B_{p+2-i}^{(k)}\right)
\end{aligned}
$$

Now we employ the notation

$$
B_{n}(x)=(B+x)^{n}, \quad B_{n}^{(k)}(x)=\left(B^{(k)}+x\right)^{n} \quad(n \geq 0) .
$$

Assume that $h, m$ are relatively prime positive integers. Then we see that

$$
\begin{aligned}
& m^{p} \sum_{\mu=0}^{m-1} \sum_{s=0}^{p+1}\left(\begin{array}{c}
p+1 \\
s
\end{array}\right) h^{s} B_{s}^{(k)}(\mu / m) B_{p+1-s}(h-[h \mu / m]) \\
& =m^{p} \sum_{\mu=0}^{m-1} \sum_{s=0}^{p+1}\left(\begin{array}{c}
p+1 \\
s
\end{array}\right) h^{s}\left(B^{(k)}+\mu m^{-1}\right)^{s}(B+h-[h \mu / m])^{p+1-s} \\
& =m^{p} \sum_{\mu=0}^{m-1}\left(h B^{(k)}+h \mu m^{-1}+B+h-[h \mu / m]\right)^{p+1} \\
& =m^{p} \sum_{\mu=0}^{m-1}\left(h B^{(k)}+h+B+\frac{1}{2}+\frac{h \mu}{m}-[h \mu / m]-\frac{1}{2}\right)^{p+1} \\
& =m^{p} \sum_{\mu=0}^{m-1}\left(h B^{(k)}+h+B+\frac{1}{2}+\bar{B}_{1}(h \mu / m)\right)^{p+1} \cdot
\end{aligned}
$$

Now, as the index $\mu$ ranges over the values $\mu=0,1,2, \ldots, m-1$, the product $h \mu$ ranges over a complete residue system modulo $m$, and due to the periodicity of $\bar{B}_{1}(x)$, the term $\bar{B}_{1}(h \mu / m)$ may be replaced by $\bar{B}_{1}(\mu / m)$ without altering the sum over $\mu$. Thus the sum 
(32) is equal to

$$
\begin{aligned}
m^{p} & \sum_{m=0}^{m-1}\left(h B^{(k)}+h+B+\frac{1}{2}+\bar{B}_{1}\left(\frac{\mu}{m}\right)\right)^{p+1} \\
& \left.=m^{p} \sum_{m=0}^{m-1}\left(h\left(B^{(k)}+1\right)+B+\frac{\mu}{m}\right)\right)^{p+1} \\
& =m^{p} \sum_{\mu=0}^{m-1} \sum_{s=0}^{p+1}\left(\begin{array}{c}
p+1 \\
s
\end{array}\right)\left(B+\frac{\mu}{m}\right)^{s} h^{p+1-s}\left(B^{(k)}+1\right)^{p+1-s} \\
& =m^{p} \sum_{\mu=0}^{m-1} \sum_{s=0}^{p+1}\left(\begin{array}{c}
p+1 \\
s
\end{array}\right) B_{s}\left(\frac{\mu}{m}\right) h^{p+1-s} B_{p+1-s}^{(k)}(1) \\
& =\sum_{s=0}^{p+1}\left(\begin{array}{c}
p+1 \\
s
\end{array}\right) m^{s-1} \sum_{\mu=0}^{m-1} B_{s}\left(\frac{\mu}{m}\right)(m h)^{p+1-s} B_{p+1-s}^{(k)}(1) \\
& =\sum_{s=0}^{p+1}\left(\begin{array}{c}
p+1 \\
s
\end{array}\right) B_{s}(m h)^{p+1-s} B_{p+1-s}^{(k)}(1),
\end{aligned}
$$

where we used the fact (a) in Lemma 1.

Therefore we obtain the following theorem.

Theorem 8 For $m, n, h \in \mathbb{N}$ with $(h, m)=1$ and any positive odd integer $p \geq 3$, we have

$$
\begin{aligned}
& \sum_{s=0}^{p+1}\left(\begin{array}{c}
p+1 \\
s
\end{array}\right) B_{s} B_{p+1-s}^{(k)}(1)(m h)^{p+1-s} \\
& \quad=m^{p} \sum_{\mu=0}^{m-1} \sum_{s=0}^{p+1}\left(\begin{array}{c}
p+1 \\
s
\end{array}\right) h^{s} B_{s}^{(k)}(\mu / m) B_{p+1-s}\left(h-\left[\frac{h \mu}{m}\right]\right) .
\end{aligned}
$$

Now we observe that

$$
\begin{aligned}
\sum_{n=0}^{\infty} B_{n}^{(k)}(x) \frac{t^{n}}{n !} & =\frac{\operatorname{Ei}_{k}(\log (1+t))}{e^{t}-1} e^{x t}=\frac{\operatorname{Ei}_{k}(\log (1+t))}{e^{d t}-1} \sum_{i=0}^{d-1} e^{(i+x) t} \\
& =\frac{\operatorname{Ei}_{k}(\log (1+t))}{d t} \sum_{i=0}^{d-1} e^{(i+x) t} \frac{d t}{e^{d t}-1} \\
& =\sum_{j=0}^{\infty} d^{j-1} \sum_{i=0}^{d-1} B_{j}\left(\frac{x+i}{d}\right) \frac{t^{j}}{j !} \frac{1}{t} \sum_{l=1}^{\infty} \frac{(\log (1+t))^{l}}{(l-1) ! l^{k}} \\
& =\sum_{j=0}^{\infty} d^{j-1} \sum_{i=0}^{d-1} B_{j}\left(\frac{x+i}{d}\right) \frac{t^{j}}{j !} \frac{1}{t} \sum_{l=1}^{\infty} \frac{1}{l^{k-1}} \sum_{m=l}^{\infty} S_{1}(m, l) \frac{t^{m}}{m !} \\
& =\sum_{j=0}^{\infty} d^{j-1} \sum_{i=0}^{d-1} B_{j}\left(\frac{x+i}{d}\right) \frac{t^{j}}{j !} \frac{1}{t} \sum_{m=1}^{\infty} \sum_{l=1}^{m} \frac{S_{1}(m, l)}{l^{k-1}} \frac{t^{m}}{m !} \\
& =\sum_{j=0}^{\infty} d^{j-1} \sum_{i=0}^{d-1} B_{j}\left(\frac{x+i}{d}\right) \frac{t^{j}}{j !} \sum_{m=0}^{\infty} \sum_{l=1}^{m+1} \frac{S_{1}(m+1, l)}{l^{k-1}(m+1)} \frac{t^{m}}{m !}
\end{aligned}
$$




$$
=\sum_{n=0}^{\infty}\left(\sum_{j=0}^{n} \sum_{i=0}^{d-1} \sum_{l=1}^{n-j+1}\left(\begin{array}{l}
n \\
j
\end{array}\right) d^{j-1} B_{j}\left(\frac{x+i}{d}\right) \frac{S_{1}(n-j+1, l)}{(n-j+1) l^{k-1}}\right) \frac{t^{n}}{n !}
$$

where $d$ is a positive integer.

Therefore by comparing the coefficients on both sides of (34) we obtain the following theorem.

Theorem 9 For $k \in \mathbb{Z}, d \in \mathbb{N}$, and $n \geq 0$, we have

$$
B_{n}^{(k)}(x)=\sum_{j=0}^{n} \sum_{i=0}^{d-1} \sum_{l=1}^{n-j+1}\left(\begin{array}{l}
n \\
j
\end{array}\right) d^{j-1} B_{j}\left(\frac{x+i}{d}\right) \frac{S_{1}(n-j+1, l)}{(n-j+1) l^{k-1}} .
$$

From (25), using Theorem 9 and (c) in Lemma 1, we see that

$$
\begin{aligned}
& h m^{p} S_{p}^{(k)}(h, m)+m h^{p} S_{p}^{(k)}(m, h) \\
& =h m^{p} \sum_{\mu=0}^{m-1} \frac{\mu}{m} \bar{B}_{p}^{(k)}\left(\frac{h \mu}{m}\right)+m h^{p} \sum_{\nu=0}^{h-1} \frac{v}{h} \bar{B}_{p}^{(k)}\left(\frac{m v}{h}\right) \\
& =h m^{p} \sum_{\mu=0}^{m-1} \frac{\mu}{m} \sum_{j=0}^{p} h^{j-1}\left(\begin{array}{c}
p \\
j
\end{array}\right) \sum_{\nu=0}^{h-1} \sum_{l=1}^{p-j+1} \frac{S_{1}(p-j+1, l)}{(p-j+1) l^{k-1}} \bar{B}_{j}\left(\frac{\mu}{m}+\frac{v}{h}\right) \\
& +m h^{p} \sum_{\nu=0}^{h-1} \frac{v}{h} \sum_{j=0}^{p} m^{j-1}\left(\begin{array}{l}
p \\
j
\end{array}\right) \sum_{\mu=0}^{m-1} \sum_{l=1}^{p-j+1} \frac{S_{1}(p-j+1, l)}{(p-j+1) l^{k-1}} \bar{B}_{j}\left(\frac{v}{h}+\frac{\mu}{m}\right) \\
& =\sum_{\mu=0}^{m-1} \frac{\mu}{m} \sum_{j=0}^{p} m^{p-j}(m h)^{j}\left(\begin{array}{l}
p \\
j
\end{array}\right) \sum_{\nu=0}^{h-1} \sum_{l=1}^{p-j+1} \bar{B}_{j}\left(\frac{\mu}{m}+\frac{v}{h}\right) \frac{S_{1}(p-j+1, l)}{(p-j+1) l^{k-1}} \\
& +\sum_{\nu=0}^{h-1} \frac{v}{h} \sum_{j=0}^{p} h^{p-j}(m h)^{j}\left(\begin{array}{l}
p \\
j
\end{array}\right) \sum_{\mu=0}^{m-1} \sum_{l=1}^{p-j+1} \bar{B}_{j}\left(\frac{v}{h}+\frac{\mu}{m}\right) \frac{S_{1}(p-j+1, l)}{(p-j+1) l^{k-1}} \\
& =\sum_{\mu=0}^{m-1} \sum_{j=0}^{p} \sum_{\nu=0}^{h-1} \sum_{l=1}^{p-j+1}(\mu h)(m h)^{-1} m^{p-j}(m h)^{j}\left(\begin{array}{l}
p \\
j
\end{array}\right) \bar{B}_{j}\left(\frac{\mu}{m}+\frac{v}{h}\right) \frac{S_{1}(p-j+1, l)}{(p-j+1) l^{k-1}} \\
& +\sum_{\mu=0}^{m-1} \sum_{j=0}^{p} \sum_{\nu=0}^{h-1} \sum_{l=1}^{p-j+1}(m v)(m h)^{-1} h^{p-j}(m h)^{j}\left(\begin{array}{c}
p \\
j
\end{array}\right) \bar{B}_{j}\left(\frac{v}{h}+\frac{\mu}{m}\right) \frac{S_{1}(p-j+1, l)}{(p-j+1) l^{k-1}} \\
& =\sum_{\mu=0}^{m-1} \sum_{j=0}^{p} \sum_{\nu=0}^{h-1} \sum_{l=1}^{p-j+1} \frac{(m h)^{j-1}\left(\begin{array}{l}
p \\
j
\end{array}\right) S_{1}(p-j+1, l)}{(p-j+1) l^{k-1}}\left((\mu h) m^{p-j}+(m \nu) h^{p-j}\right) \bar{B}_{j}\left(\frac{v}{h}+\frac{\mu}{m}\right) .
\end{aligned}
$$

Therefore we obtain the following reciprocity relation.

Theorem 10 For $m, h, p \in \mathbb{N}$ and $k \in \mathbb{Z}$, we have

$$
\begin{aligned}
& h m^{p} S_{p}^{(k)}(h, m)+m h^{p} S_{p}^{(k)}(m, h) \\
& \quad=\sum_{\mu=0}^{m-1} \sum_{j=0}^{p} \sum_{\nu=0}^{h-1} \sum_{l=1}^{p-j+1} \frac{(m h)^{j-1}\left(\begin{array}{c}
p \\
j
\end{array}\right) S_{1}(p-j+1, l)}{(p-j+1) l^{k-1}}\left((\mu h) m^{p-j}+(m \nu) h^{p-j}\right) \bar{B}_{j}\left(\frac{v}{h}+\frac{\mu}{m}\right) .
\end{aligned}
$$


In the case $k=1$, we obtain the following reciprocity relation for the generalized Dedekind sum defined by Apostol.

Corollary 11 For $m, h, p \in \mathbb{N}$, we have

$$
\begin{aligned}
h m^{p} & S_{p}(h, m)+m h^{p} S_{p}(m, h) \\
& =\sum_{\mu=0}^{m-1} \sum_{\nu=0}^{h-1}(m h)^{p-1}(\mu h+m v) \bar{B}_{p}\left(\frac{v}{h}+\frac{\mu}{m}\right) \\
& =(m h)^{p} \sum_{\mu=0}^{m-1} \sum_{v=0}^{h-1}(m h)^{-1}(\mu h+m v) \bar{B}_{p}\left(\frac{v}{h}+\frac{\mu}{m}\right) .
\end{aligned}
$$

\section{Conclusion}

The Dedekind sums are defined by

$$
S(h, m)=\sum_{\mu=1}^{m}\left(\left(\frac{\mu}{m}\right)\right)\left(\left(\frac{h \mu}{m}\right)\right) \quad(\text { see }[1,4,6-8,11-13]) .
$$

In 1952, Apostol considered the generalized Dedekind sums and introduced interesting and important identities and theorems related to his generalized Dedekind sums. These Dedekind sums are a field studied by various researchers. Recently, the modified Hardy polyexponential function of index $k$ is introduced by

$$
\operatorname{Ei}_{k}(x)=\sum_{n=1}^{\infty} \frac{x^{n}}{n^{k}(n-1) !}, \quad(k \in \mathbb{Z})(\text { see }[5,9])
$$

In [5] the type 2 poly-Bernoulli polynomials of index $k$ are defined in terms of the polyexponential function of index $k$ by

$$
\frac{\operatorname{Ei}_{k}(\log (1+t))}{e^{t}-1} e^{x t}=\sum_{n=0}^{\infty} B_{n}^{(k)}(x) \frac{t^{n}}{n !} \quad(k \in \mathbb{Z}) .
$$

In this paper, we thought of the poly-Dedekind sums from the perspective of the Apostol generalized Dedekind sums. That is, we considered the poly-Dedekind sums derived from the type 2 poly-Bernoulli functions and polynomials.

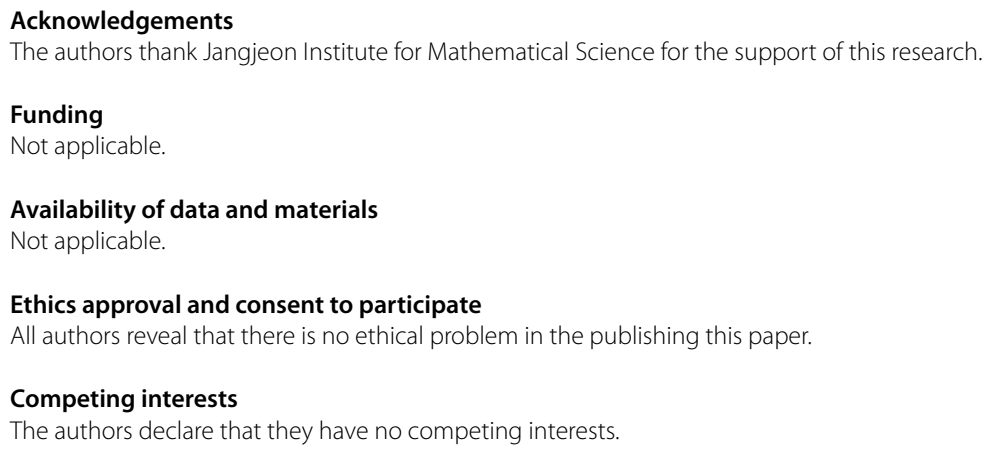


Consent for publication

All authors want to publish this paper in this journal.

\section{Authors' contributions}

TK and DSK conceived of the framework and structured the whole paper; TK and DSK wrote the paper; L-CJ and HL checked the results of the paper; DSK and TK completed the revision of the paper. All authors have read and agreed to the published version of the manuscript.

\section{Author details}

'Department of Mathematics, Kwangwoon University, Seoul 139-701, Republic of Korea. ${ }^{2}$ Department of Mathematics, Sogang University, Seoul 121-742, Republic of Korea. ${ }^{3}$ Graduate School of Education, Konkuk University, Seoul, Republic of Korea.

\section{Publisher's Note}

Springer Nature remains neutral with regard to jurisdictional claims in published maps and institutional affiliations.

Received: 10 September 2020 Accepted: 1 October 2020 Published online: 07 October 2020

\section{References}

1. Apostol, T.M.: Theorems on generalized Dedekind sums. Pac. J. Math. 2, 1-9 (1952)

2. Bayad, A.: On values of Jacobi forms and elliptic Dedekind sums. Proc. Jangjeon Math. Soc. 12(2), 119-139 (2009)

3. Can, M., Cenkci, M., Kurt, V., Simsek, Y.: Twisted Dedekind type sums associated with Barnes' type multiple Frobenius-Euler I-functions. Adv. Stud. Contemp. Math. (Kyungshang) 18(2), 135-160 (2009)

4. Carlitz, L.: Some theorems on generalized Dedekind-Rademacher sums. Pac. J. Math. 75(2), 347-358 (1978)

5. Kim, D.S., Kim, T.: A note on polyexponential and unipoly functions. Russ. J. Math. Phys. 26(1), 40-49 (2019)

6. Kim, T:: A note on $p$-adic $q$-Dedekind sums. C. R. Acad. Bulgare Sci. 54(10), 37-42 (2001)

7. Kim, T.: Note on Dedekind type DC sums. Adv. Stud. Contemp. Math. (Kyungshang) 18(2), 249-260 (2009)

8. Kim, T.: Note on q-Dedekind-type sums related to q-Euler polynomials. Glasg. Math. J. 54(1), 121-125 (2012)

9. Kim, T., Kim, D.S.: Degenerate polyexponential functions and degenerate Bell polynomials. J. Math. Anal. Appl. 487(2), $124017(2020)$

10. Kim, T., Kim, D.S., Kwon, J., Lee, H.: Degenerate polyexponential functions and type 2 degenerate poly-Bernoulli numbers and polynomials. Adv. Differ. Equ. 2020, 168 (2020)

11. Simsek, Y:: Remarks on reciprocity laws of the Dedekind and Hardy sums. Adv. Stud. Contemp. Math. (Kyungshang) 12(2), 237-246 (2006)

12. Simsek, Y:: q-Dedekind type sums related to $q$-zeta function and basic L-series. J. Math. Anal. Appl. 318(1), 333-351 (2006)

13. Simsek, Y.: Special functions related to Dedekind-type DC-sums and their applications. Russ. J. Math. Phys. 17(4), 495-508 (2010)

\section{Submit your manuscript to a SpringerOpen ${ }^{\circ}$ journal and benefit from:}

- Convenient online submission

- Rigorous peer review

- Open access: articles freely available online

- High visibility within the field

- Retaining the copyright to your article 